\title{
Dynamical Image-based PID Uncalibrated Visual Servoing with Fixed Camera for Tracking of Planar Robots with a Heuristical Predictor
}

\author{
$\nmid$ E.C. Dean-León, $\ddagger V$. Parra-Vega, $\S$ A.Espinosa-Romero and $\dagger J$. Fierro \\ †Mechatronics Division - CINVESTAV, México, \{edean,jfierro\}@mail.cinvestav.mx \\ $\ddagger$ Center for Advanced Technology - CIATEQ, Retablo 150, Fovissste, Querétaro, Qro., México \\ $\S$ Facultad de Matemáticas, Universidad Autónoma de Yucatán, México, eromero@tunku.uady.mx.
}

\begin{abstract}
Based on a recent state-of-the-art image-based visual feedback control scheme in fixed uncalibrated camera proposed by the authors [3], the experimental verification is presented. However, image acquisition and processing for closed-loop control introduces technological challenges through the time delay induced by the CCD camera itself. Thus, to preserve the time domain algorithm and to avoid discretization of our nonlinear controller, a practical predictor is introduced to obtain rough estimates of the image at the bandwidth of the robot. The nonlinear dynamics of the planar robot is considered for the stability analysis. Exponential tracking arises due to a chattering-free visual sliding mode using PID controller based on image error trajectories. The experimental setup describes details of the implementation of our previous theoretical results using a FireWire fixed camera, and a direct drive robot for desired image trajectories. All camera and robot parameters are unknown, and experimental results confirms the theoretical results.
\end{abstract}

Index Terms - Visual Servoing, Nonlinear PID Control, Robot Manipulators, Predictor

\section{INTRODUCTION}

Research on kinematic-based visual servoing for robots considers the robots as serial kinematic chain, with any dynamics involved, of course. In this way, the output of the kinematic-based visual servoing schemes stand for the desired cartesian velocity for a given dynamical robot controller. The underlying assumption of these schemes is that there exists a very fast dynamical controller that yields exactly the desired cartesian velocity at every instant, otherwise there will be mismatch in the differential kinematic mapping. Since a given dynamical controller cannot produce exactly the desired cartesian velocity at every instant, then it violates the whole formulation of kinematic-based visual servoing. However, to keep the formulation as physically valid, feedback gains are manipulated so as to very slow desired cartesian velocity are produced, and then the dynamical robot controller has more chance to track more precisely this trajectory. The result is a low performance visual servoing scheme.

An alternative approach to deal with high performance visual servoing is to take into account explicitly the dynamics. These approach has recently been tackled by a few authors. The result is an explicit computation of the dynamical robot controller, that can track desired trajectories, not necessarily low velocity trajectories. That is, dynamical visual servoing schemes considers dynamical robot manipulators, not serial kinematic chains as does kinematic-based visual servoing.

In contrast to the large number of approaches in kinematic-based visual servoing, very few schemes are available for dynamic-based visual servoing. Uncalibrated camera with unknown robot parameters are considered in [1], [2], [5], [6]. Adaptive control techniques guarantee asymptotic stability with a considerable load on the online computation of the regressor and adaptive parameters. In contrast, [3] presents a regressor free controller which guarantees exponential tracking in image space in fixed camera configuration for $2 \mathrm{D}$ image-based tracking.

\section{A. Contribution}

The real time performance in the continuous domain of the novel dynamical visual servoing scheme [3] is presented and discussed. This is possible due to a proposed predictor based on heuristical hypothesis of the Smith Predictor. The salient feature is the simplicity of the decentralized controller, which exhibits a PID structure based on image tracking errors. A rough estimation of the image jacobian and camera parameters. Experiments show that even under $99 \%$ of uncertainty of these parameters, exponential tracking is obtained. A predictor is proposed to deal with the multirating nature of the visual servoing scheme. This structure is discussed and experimentally validated.

\section{Il. ROBOT MODEL}

The dynamics of a serial $n$-link rigid, non-redundant, fully actuated robot manipulator can be written as follows

$$
H(q) \ddot{q}+C(q, \dot{q}) \dot{q}+G(q)=\tau-f_{r}
$$

where $q \in R^{n}$ is the vector of generalized joint displacements, with $\dot{q} \in R^{n}$ its velocity, $\tau \in R^{n \times 1}$ stands for the vector applied joint torques, $H(q) \in R^{n \times n}$ is the symmetric positive definite manipulator inertia matrix, $C(q, \dot{q}) \dot{q} \in R^{n}$ stands for the vector of centripetal and Coriolis torques, $G(q) \in R^{n}$ is the vector of gravitational torques, and finally $f_{\mathrm{r}}=B_{0} \dot{q} \in R^{n}$ stands for the viscous forces, wherein $B_{0} \in R^{n \times n}$ is the coefficient of viscous forces. 


\section{CAMERA Model}

For planar robots, and using thin lens without aberration, [4] presented the widely accepted fixed (static) camera configuration, whose basic mathematical description of this system consists of a composition of four transformations defined as follows

- Joint to Cartesian transformation

- Cartesian to Camera transformation

- Camera to CCD transformation

- CCD to Image transformation

Then the following inverse differential kinematics that relates generalized joint velocities $\dot{q}$ and image velocities $\dot{x}$ appears

$$
\dot{q}=J^{-1} R^{-1} \alpha^{-1} \dot{x} \equiv J^{-1} R_{\alpha}^{-1} \dot{x}
$$

where $\alpha$ is the scaling factor, $J(q)$ is the analytic Jacobian matrix of the manipulator, $R_{\alpha}^{-1}=(\alpha R)^{-1}, x \in R^{2}$ stands for the end-effector transformation from robot base to camera base. In the screen coordinate frame, the robot position is captured with the following position, given in pixels,

$$
\begin{aligned}
x & =\alpha_{0} \frac{\lambda_{f}}{\lambda_{f}-z}\left[\begin{array}{cc}
-1 & 0 \\
0 & 1
\end{array}\right] R_{0} f(q)+\beta \\
& =\alpha R f(q)+\beta
\end{aligned}
$$

where $\alpha_{0}$ is the scaling parameter ${ }^{1}, R_{0} \in R^{2 \times 2}$ stands for the $2 \times 2$ upper square matrix of $R_{\theta} \in S O(3)$, that is

$$
\begin{aligned}
R_{0} & =\left[\begin{array}{cc}
-\cos (\theta) & \sin (\theta) \\
\sin (\theta) & \cos (\theta)
\end{array}\right] \\
\alpha & =\alpha_{0} \frac{\lambda_{f}}{\lambda_{f}-z}<0 \\
R & =\left[\begin{array}{cc}
\cos (\theta) & -\sin (\theta) \\
\sin (\theta) & \cos (\theta)
\end{array}\right] \\
\beta & =\alpha_{0} \frac{\lambda_{f}}{\lambda_{f}-z}\left[\begin{array}{l}
{ }^{v} O_{b 1} \\
{ }^{v} O_{b 2}
\end{array}\right]+\left[\begin{array}{l}
u_{c} \\
v_{c}
\end{array}\right]
\end{aligned}
$$

where $\lambda_{f}$ is the focal length along the optical axis, $z$ stands for the depth of field, $u_{c}, v_{c}$ stands for the cartesian position in image coordinates, and ${ }^{v} O_{b 1},-{ }^{v} O_{b 2}$ stands for the offset between optical axis and $\vec{Z}$ of the robot base

\section{Dynamic Visual Servoing Problem}

There are some fundamental problems in visual servoing tasks. The scientific problem is that visual servoing poses a rather ill-problem since the variable to control is not a dynamic, that is the pose of the robot in image coordinates is static variable, it depends on a static mapping from joint coordinates. There is not a control variable in image coordinates, image coordinates does not stand as an state of the system. Also the closed loop system is in fact two-time scale system.

On the other hand, the technological problem is that the servo loop of the robot control system runs much faster than the refreshing rate of the image sensor, the CCD camera, which usually is. say $33 \mathrm{~Hz}$ and the sampling rate of the robot is, say $1 \mathrm{~K} \mathrm{~Hz}$. So there arise problems with

${ }^{1}$ Without loss of generality, $\alpha_{0}$ can be considered as a $2 \times 2$ scaling matrix. the latency of the camera, which are typically overlooked and if there are not addressed properly, it is quite easy to prove instability.

In this paper, we consider that a predictor delivers an estimate of the image at the same rate of the sampling period of the servo loop of the robot, that is at $1 \mathrm{~Hz}$. In this way, we preserve only one sampling period and we reasonably assume that this is fast enough to consider a time-varying formulation, rather a discrete one. And we consider passivity based approach to design a controller for the following problem.

Design a smooth joint torque robot control input for an uncalibrated camera and unknown full nonlinear robot dynamics (regressor is not available), such that the closed loop system guarantees image-based trajectory tracking.

The previous statement poses some new issues into the dynamic visual servoing problem: apart from kinematicbased visual servoing [4], wherein robot dynamics are ignored, dynamic-based visual servoing makes use of the model of the system through the regressor [1], [2], [5], [6]. In this paper. we are concerned to the experimental validation of ([3]) wherein the regressor is not required, which is usually the case for industrial robots since the exact regressor is hardly available.

\section{A. Structure of the proposed controller}

The fix camera is modeled as a static operator (3) that relates position and velocities of image and joint coordinates. Then, we are interested in designing an uncalibrated joint output error manifold $\bar{s}_{q}$ related to a measurable visual error manifold $s_{x}$, such that establishment of a passivity inequality for $\left\langle\vec{s}_{q}, \tau^{*}\right\rangle$ implies dissipativity for output $s_{x}$. This means that if we can find $\tau^{*}$ independently of the regressor, then $s_{x}$ converges, and the controller will satisfy the problem given above. To this end, we need to derive the robot dynamics in $\bar{s}_{q}$ coordinates, such that the passivity inequality dictates the control structure as well as the storage function. Doing so will allow us to explore some known techniques in passivity-based robot control. Now, how can we implement this control system? The continuous domain controller is very powerful, in a sense that delivers high performance tracking (simple control structure, low computational cost and fast tracking regime), thus we would like to preserve this structure. Therefore. we need to introduce somehow a predictor which delivers estimated images at faster rate, ideally, also at $1 \mathrm{KHz}$. To this end, in this paper, we present an predictor that allows to obtain all signals at the same rate, and the real time performance yields the expected stability properties of the controller.

\section{DYNAMiC UnCalibrated Visual SERVOING}

Consider the following definitions [3]

$$
\dot{\bar{q}}_{r}=\bar{J}^{-1} \bar{R}_{\alpha}^{-1} \dot{\bar{c}}_{r}
$$

where $\bar{J}^{-1}$ and $\bar{R}_{\alpha}^{-1}$ stand for some estimate of the inverse of the analytical jacobian $J^{-1}$ and the inverse of the modulated rotational matrix $R_{\alpha}^{-1}$, respectively. The 
nominal reference $\dot{x}_{r}$ is given by

$$
\begin{aligned}
\dot{x}_{r} & =\dot{x}_{d}-\lambda \Delta x+s_{d}-K_{i} v \\
\dot{v} & =\operatorname{sgn}\left(s_{\delta}\right)
\end{aligned}
$$

where $x_{d}$ and $\dot{x}_{d}$ denote the desired position and velocity of the end-effector with respect to the image frame, respectively, and

$$
\begin{aligned}
s_{\delta} & =s-s_{d} \\
s & =\Delta \dot{x}+\lambda \Delta x \\
s_{d} & =s\left(t_{0}\right) \exp ^{-\kappa t}
\end{aligned}
$$

with the integral feedback gain $K_{i}=K_{i}^{T} \in R_{+}^{n \times n}$ whose precise lower bound is to be defined yet; $\lambda>0 ; \kappa>$ 0 ; the $\operatorname{sgn}(y)$ is the entrywise discontinuous $\operatorname{signum}(y)$ function of $y \in R^{n} ; \Delta x=x-x_{d}$ is the image-based end-effector position tracking error; $s_{d} \in C^{1}$ and thus $s_{\delta}\left(t_{0}\right)=0 \forall t$. Now, we are ready to state the main result [3]

Theorem 1: Consider a robot manipulator (1) in closed loop with the following decentralized sliding PID visual servoing scheme

$$
\tau=-K_{d} \bar{s}_{q}
$$

Then. the closed-loop system yields locally exponentially $\lim _{t \rightarrow \infty} \Delta x=0, \quad \lim _{t \rightarrow \infty} \Delta \dot{x}=0$ provided that $K_{d}$ and $K_{i}$ are tuned large enough, for small enough initial error conditions.

Proof. See [3].

\section{REMARKS}

\section{A. Two control feedback loops}

It is assumed that there is a joint sensor, that is an encoder that measures $q$ and $X, \dot{X}$ are available.

\section{B. Two control feedback loops}

The stability analysis suggest that a damping force equivalent to $K_{d} \bar{s}_{q} N m$ is implemented to stabilize the system around the uncalibrated joint error manifold $\bar{s}_{q}=$ 0 . On the other hand, $\dot{v}=\operatorname{sgn}\left(s_{\delta}\right)$ switches over the visual error manifold $s_{\delta}=0$ to induce precisely a sliding mode at $s_{\delta}=0$, to finally converge toward its equilibrium $\Delta x=0$, and $\Delta \dot{x}=0$.

\section{PlD-like structure}

The controller (14) can be written as follows

$$
\begin{aligned}
& \tau=-K_{p}(t) \Delta x-K_{v}(t) \Delta \dot{x}-\bar{K}_{i} I+\Sigma_{12}(t) \\
& \dot{I}=\operatorname{sgn}\left(s_{\delta}\right)
\end{aligned}
$$

where $\Sigma_{12}(t)=\Sigma_{1}(t)+\Sigma_{2}(t)$ and

$$
\begin{aligned}
K_{p}(t) & =K_{d} J^{-1} R_{\alpha}^{-1} \lambda \\
K_{v}(t) & =K_{d} J^{-1} R_{\alpha}^{-1} \\
\bar{K}_{i} & =K_{d} K_{i} \Sigma_{1}(t)
\end{aligned}
$$$$
=K
$$

$\Sigma_{1}(t)$ is useful to obtain the sliding mode and $\Sigma_{2}(t)=$ compensates for the uncertainty of camera parameters.

$$
\begin{aligned}
\tau & =-K_{d} \bar{s}_{q} \equiv-K_{d}\left\{s_{q}-Y_{v} \Delta \theta_{v}\right\} \\
& =-K_{d}\left(J ^ { - 1 } R _ { \alpha } ^ { - 1 } \left\{\Delta \dot{x}+\lambda \Delta x-s\left(t_{0}\right) \exp ^{-\kappa t}+\right.\right. \\
& \left.\left.K_{i} \int_{t 0}^{t} \operatorname{sgn}\left(s_{\delta}\right)\right\}-K_{d} Y_{c} \Delta_{v} \theta_{v}\right)
\end{aligned}
$$

D. Control structure

It is evident the simple structure of this controller, only an estimate of the composition of the image jacobian, the rotation matrix and the scaling and depth parameters are required.

\section{E. Robust tracking}

The stability analysis of the theorem shows that a second order sliding mode is induced, and thus chatteringfree control effort arises with robust image-based tracking, typical of variable structure controllers.

\section{F. $3 D$ visual servoing?}

Our control scheme is valid only for the planar case, but if the jacobian is available, this algorithm can be extended to $n \geq 3$ degrees of freedom simply by using a proper camera projection model, and substituting the real jacobian by the estimate one in the controller.

VII. A Practical Solution to the Multiple

Time-sCale Problem of Visual SERVoing

The system is in reality a two time scales system, and the latency of the camera is not negligible. Therefore, for control theory researchers, it seems that the correct formulation is a discrete formulation together with twotime-scale-system theory, such as singularity perturbed systems. Those can render a very complex formulation, only reachable by control theorists and manipulate to grasp by robotics researchers. Thus, the plausible solution is to preserve the time domain rather that discrete domain at the expenses of ensure a unique fast sampling period of all closed loop signals.

To do this, en this paper we need to obtain the image at $1 \mathrm{~ms}$. Taking the idea of prediction techniques, we design a heuristic predictor derived from image based robot end effector task. Once we have induced the second order sliding mode in the system, this implies local exponential convergence of image based visual tracking errors, as we probe in last sections. In this way, we can say, for some $\Delta x, \Delta \dot{x} \in[0, \varepsilon]$, where $\varepsilon$ is a image based position and velocity small error, a second order sliding mode has been induced and this error converge exponentially to zero. Using this assumption, the most probably value for robot end effector position and velocity is close to desire image based robot end effector task, this supposition can be made because the robot end effector task is designed in image space, so we can plan this task as close as we need to the robot end effector. Therefore, we use the position and velocity obtained from image based task as the current robot end effector image based position and velocity ${ }^{2}$. $\theta_{\text {This is, }}$

${ }^{2}$ This implies certain ertor between, the real position and velocity, and the estimated ones, but all prediction techniques use uncertainty factors. 


$$
\begin{aligned}
& x(k)=x_{d}(k-1), \\
& \dot{x}(k)=\dot{x}_{d}(k-1),
\end{aligned}
$$

with

$$
\begin{aligned}
& x(t)=\left\{\begin{array}{ll}
x_{r}(k), & \text { if } \bmod \left(k, s_{r}\right)=0 \\
x_{d}(k-1), & \text { if } \bmod \left(k, s_{r}\right) \neq 0
\end{array},\right. \\
& \dot{x}(t)=\left\{\begin{array}{ll}
\dot{x}_{r}(k), & \text { if } \bmod \left(k, s_{r}\right)=0 \\
\dot{x}_{d}(k-1), & \text { if } \bmod \left(k, s_{r}\right) \neq 0
\end{array},\right.
\end{aligned}
$$

where we define $x(k), \dot{x}(k)$ as image based position and velocity end effector at instant $k ; x_{d}(k-1), \dot{x}_{d}(k-1)$, as image based desired robot end effector position and velocity, deduced from image based task at instant $k-1$; $x_{r}(k), \dot{x}_{r}(k)$, as image based position and velocity end effector obtained from digital information of camera at instant $k$, with $k=0,1, \ldots, n$ the sampling rate of robot control loop and $s_{r}=1,33,66, \ldots$, is the sampling rate of vision system.

This predictor can be implemented only when $\Delta x$, $\Delta \dot{x} \in[0, \varepsilon]$. But in order to use this predictor with out the restriction of previous knowledge of $\varepsilon$, we propose a different prediction value for image based error. This new value includes the last instant position, and velocity error as follows

$$
\begin{aligned}
& \Delta x(t)=x(t)-x_{d}(t)+\Delta x(t-1), \\
& \Delta \dot{x}(t)=\dot{x}(t)-\dot{x}_{d}(t)+\Delta \dot{x}(t-1),
\end{aligned}
$$

Using this predictor of imaged based position and velocity error, we solve the multiple sampling rate problem ${ }^{3}$, implied in all most visual servoing systems. This predictor show attractive advantages because its simple and low computational cost implementation, compared with the predictors showed in [7].

\section{Simulations}

A two-rigid link, planar robot is considered, with a CCD camera, see parameters in Tables I and II. All inertial parameters of the robot arm and all parameters of the camera, including depth of field of view, are unknown. The endpoint of the manipulator is requested to draw a circle in image space defined with respect to the vision frame $y_{d}=\left(y_{d 1}, y_{d 2}\right)^{T}=(0.1 \cos \omega t+0.25,0.1 \sin \omega t+$ 0.15 ), where $\omega=0.5 \mathrm{rad} / \mathrm{sec}$. Simulations are carried in Matlab 6.0, with RungeKutta45 as the numerical solver, at $1 \mathrm{~ms}$, so it was assumed that visual data arrives also at $1 \mathrm{~ms}$, otherwise a technique to obtain $x, \dot{x}$ at $1 \mathrm{~ms}$ should be implemented, for instance prediction technique showed in section (VII) to predict the following desired trajectory. Results presented in Figure (5), shows exponential tracking capabilities of the control, with a remarkable smooth control effort.

\section{EXPERIMENTAL SYSTEM}

This system consists of tree sub-systems. These subsystems are showed in Figure (1). Figure (2) shows the tree sub-systems on fixed camera configuration.

${ }^{3}$ Resent researches have found this prediction technique stable [9].

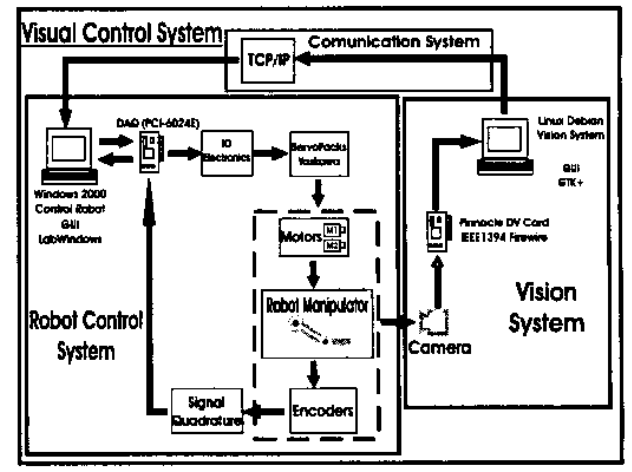

Fig. 1. Visual System

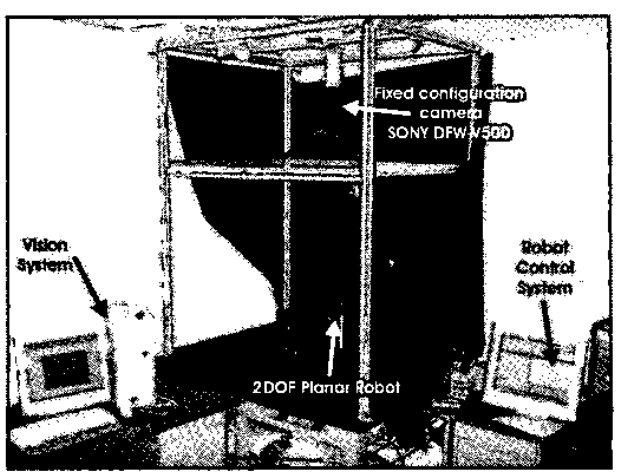

Fig. 2. Experimental System.

\section{A. Robot Control System}

1) Hardware: The 2 DOF planar robot was designed and constructed at the center. This is actuated by two $220 \mathrm{~V}$ A.C. direct drive motors with integrated optical encoders of $2048 \mathrm{ppr}$. These motors are Yaskawa SGM08A314 and SGM-04U3B4L for link 1 and 2 respectively. The drivers of motors are Yaskawa Servopacks SGD08AS and SGDA-04AS. The National Instruments PCl$6024 \mathrm{E}$ data acquisition board was used to handle the $\mathrm{V} / \mathrm{O}$ signals. In order to improve the resolution of the encoders, a hardware encoder quadrature was implemented.

2) Software: The robot control system was implemented in a $1.5 \mathrm{GHz}$ Pentium IV PC with $256 \mathrm{Mb}$ DRAM memory on Windows $2000 \mathrm{OS}$. To provide a friendly user interface, a GUI was developed on Labwindows CVI 6.0 of National Instruments.

\section{B. Vision System}

1) Hardware: The visual information is obtained with a SONY DFW-V500 digital CCD camera with $30 \mathrm{fps}$ full motion picture, which adopts the IEEE1394-1995 standard. The implementation of the vision system was done in a $1.533 \mathrm{Ghz}$ AMD $1800+$ processor, with $512 \mathrm{Mb}$ DRAM. PC. Since a real time information processing was needed, the OS selected for this station is Linux Debian 
with a Real Time Application Interface (Linux RTAI module) compiled on kernel to achieve hard real time scheduler.A Pinnacle Studio DV PC board was installed to manage the transmission of visual information through IEEE1394 ports.

2) Software: Linux provides numerous free software and applications; there exist especial libraries and modules for capture and treatment of digital visual information for the IEEE1394 standard. In order to use functions of these low level modules, we developed software libraries for image data acquisition. These libraries include functions for processing, treatment and analysis of digital images, data management functions, scheduler management functions and so on, all functions used to acquire position and velocity of the robot end effector in image space. In order to implement the visual tracking system we set up different marks to the robot end effector, robot link 1 and robot base, as showed in Figure (3). These marks were circles of different radius ${ }^{4}$.

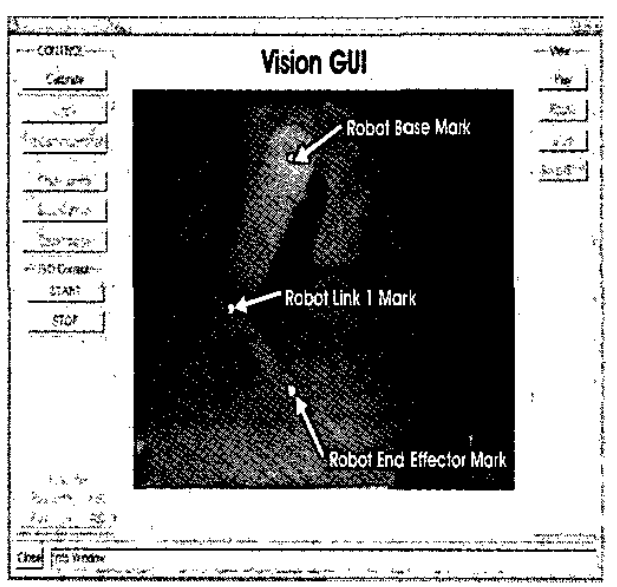

Fig. 3. Visual land marks and GUI.

The visual tracking process involve different stages. The first one is System Initialization, where image space location of the desired marks is done, using digital processing techniques as segmentation, labeling and pattern identification. These marks are characterized using image binary moments. (e.g. central moment and first order moments). The next stage is Visual Tracking, where we match and locate the selected marks in two consecutive frames and estimate their inter-frame disparity, finding the minimal mark matching error between frames, this is done using the Sum of Square Difference (SSD) as a correlation measurement [8]. This disparity is used to compute the estimated rate of change (velocity) with Euler derivation. This stage is repeated several times and, at the end of each loop, a new mark characterization is done to avoid incremental position error. the complete vision system was implemented in a hard real time.

${ }^{4}$ We selected circles as marks due to its image properties, i.e. rotational invariance.

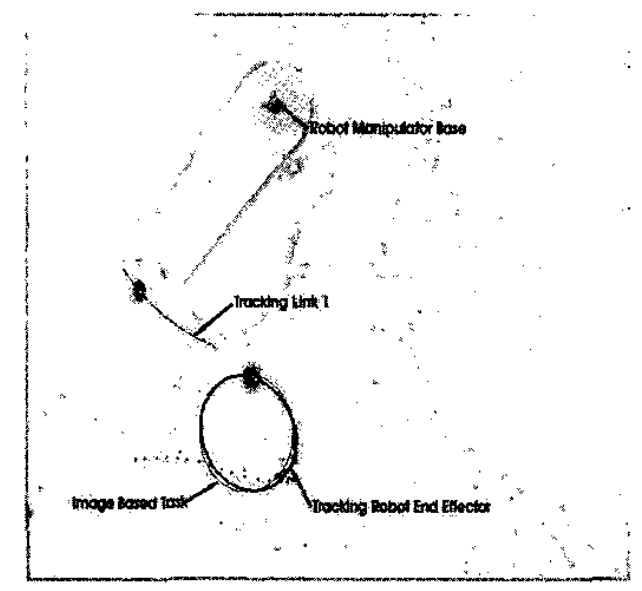

Fig. 4. The figure describes the information displayed in the GUl. A digital processing was applied to this image in order to highlight the robot end effector performance and image based task.

In addition, a user interface was coded to provide final, easy and useful, user tools (see Figure (3)). This GUI was developed in GTK+ software for Linux. The importance of this GUI is exalted due to, in this approach, robot endeffector task is designed in image space, this implies that direct access to the visual information is needed in order to define this task, and here yields the GUI importance.

\section{Communication System}

As we explain, few lines above, the experimental system consist in vision system, robot control system and communication system, the last one was created to establish a link between the other two systems. The communication is via TCP/IP protocol, the reason of this selection is simply, we have different OS for each system (Vision System-Linux, Robot Control System-Windows 2000 ), and TCP/IP protocol provides a standard language able to be used in both OS. With the aim of use this protocol, a specific function was coded in $\mathrm{C}$ language to manage information coming through this via.

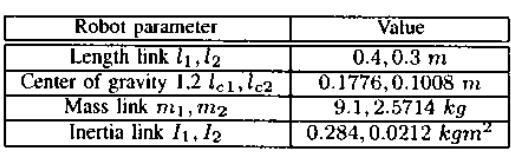

TABLE I

ROBOT PARAMETERS

\section{Experimental Resuits}

The desire image based task is follow a circle track with radius $r=40$ pixels, and centered in $\left(c_{x}, c_{y}\right)=$ $(236,355)$ pixels referenced with the image space axes. The circle is generated with an angular velocity of $\omega=$ $0.628 \mathrm{rad} / \mathrm{s}$. The position of the robot end effector is showed at the GUI in the visual system. Parameters of 


\begin{tabular}{|c|c|}
\hline Vision parameters & Value \\
\hline Clock-wise rotation angle $\theta$ & $\frac{\pi}{2} \mathrm{rad}$ \\
\hline Scale factor $\alpha_{\eta}$ & 77772 pixeles $/ \mathrm{m}$ \\
\hline Depth field of view $z$ & $1.5 \mathrm{~m}$ \\
\hline Camera offset ${ }^{v} O_{b}$ & {$[-0.2-0.1]^{T} \mathrm{~m}$} \\
\hline Offset $\Sigma_{l} o_{l}$ & {$[0.00050 .0003]^{T} \mathrm{~m}$} \\
\hline Focal length $\lambda_{f}$ & $0.008 \mathrm{~m}$ \\
\hline
\end{tabular}

TABLE II

Camera parameters
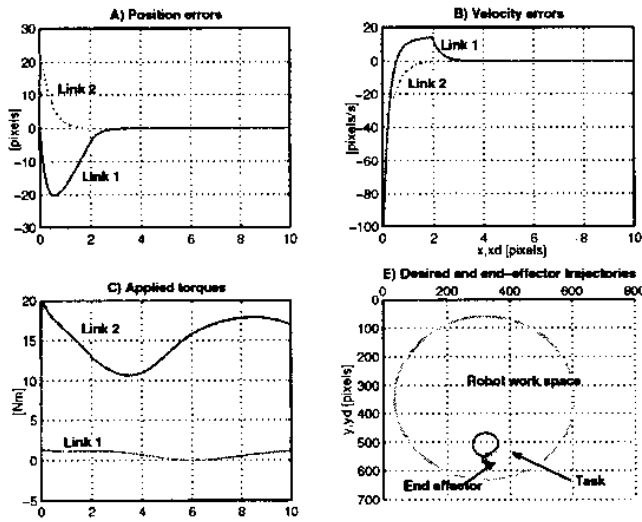

Fig. 5. Simulation Resuits

the 2 DOF planar robot and camera are showed in tables 1 and 2 . The visual field of camera covers completely the robot work space and $R_{1}-R_{2} \| S_{1}-S_{2}$, where $R_{1}-R_{2}, S_{1}-S_{2}$, stands for robot work space and image space, respectively. The intersection of optical axe with robot space is $(0.23 .0 .10) \mathrm{m}$, in terms of robot base. The capture period of camera is $30 \mathrm{fps}$. Depth of camera with respect to the robot space is $z=1.56 \mathrm{~m}$. The counterclockwise angle between image plane and robot plane is $90^{\circ}$. The focal length $\lambda=0.0076 \mathrm{~m}$.

Velocities of joint and image space were estimated using Euler derivation and filtered with second order Butterworth filter a showing next

$$
\begin{aligned}
y(k)= & b_{0} x(k)+b_{1} x(k-1)+b_{2}(k-2)- \\
& a_{1} y(k-1)-a_{2} y(k-2)
\end{aligned}
$$

where, $y$ is filtered output, $x$ is input signal, $b_{0} \ldots b_{1}$ y $a_{1}, a_{2}$, are filter coefficients and $k$ is the sampling period. The experimental results of this approach are showed in figures (6), (7), (8), (9), (10), (11), (12), (13) and (4).

\section{CONClusions}

The experimental verification of the decentralized nonlinear PID for tracking of dynamical robots using uncalibrated camera in fix configuration is presented. The controller is continuous and guarantees tracking of tasks designed in image space The robot performance, in hard real time, shows the second order visual sliding mode. The results obtained are satisfactory and more efficient than results found in literature. Tuning of control parameters

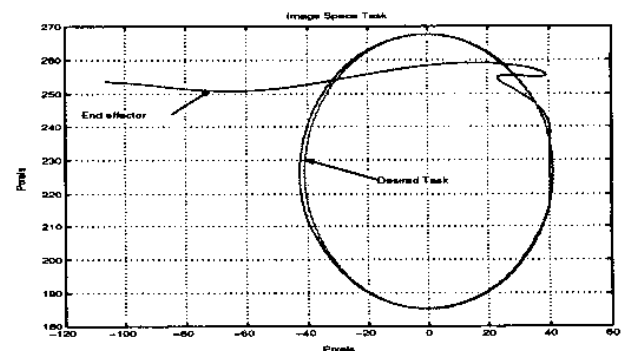

Fig. 6. In this figure is showed tracking of the task by robot end effector, as we can see the robot end effector shows excellent tracking performance. We also see in one section of tracking the position error increases, but this is just a result of a robot construction problem. This results agreed with simulation results.

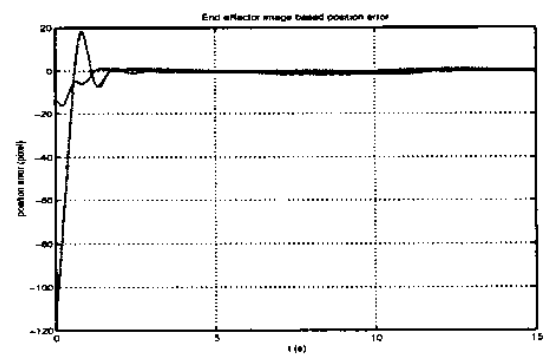

Fig. 7. This figure shows the position error convergence, this convergence is exponential, as we can see the maximum experimental image based position error presented is -2 pixels.

was achieved in experimental way. This is very easy because of its simply control law structure. The velocity of experimental system is constrained by vision system. We reached maximum angular velocity of desired task of $1.2 \mathrm{rad} / \mathrm{s}$. This scheme is valid only for $2 \mathrm{DOF}$ tasks, however it can be easily extended to the 3D case, with similar stability properties. Notice that in that case, there may appear singular configurations, similar to [6].

\section{ACKNOWLEDGEMENTS}

V. Parra-Vega carries out this research while he is on a sabbatical leave from Mechatronics Division, CINVESTAV, at the Information Technology Division of ClATEQ. This author acknowledges support from CONACYT project number $39727-Y$ and CUDI project under call for Intemet2 Applications Spring 2003.

\section{XI1. REFERENCES}

[1] Hsu, L., and Aquino, P., Adaptive Visual Tracking with Uncertain Manipulator Dynamics and Uncalibrated Camera, 1999 Proc. 38th IEEE Conference on Decision and Control, pp. 1248-1253.

[2] Bishop, B.E.. and M.W. Spong, Adaptive calibration and control of 2D monocular visual servo systems, IFAC Symp. on Robot Control, France, 1997. 


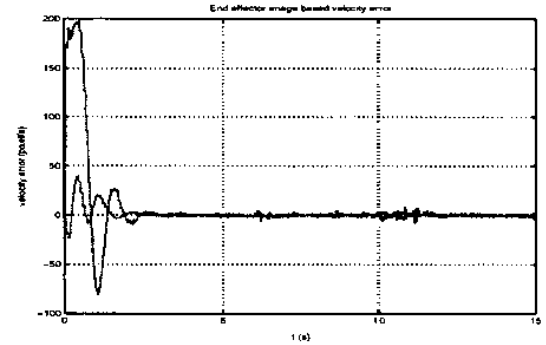

Fig. 8. The figure shows the velocity error. and we can see that this error converges to cero. The problem presented in position error (Figure (6)) is not showed in this performance.

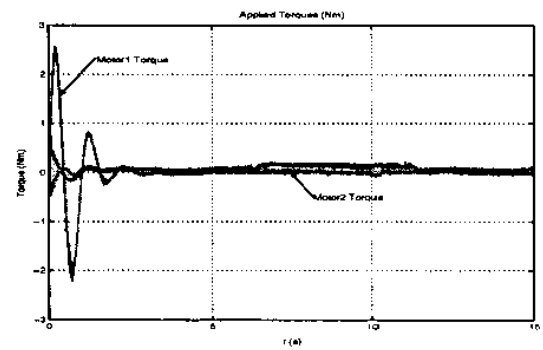

Fig. 9. We show the applied torques of planar robot actuators, is easy to observe that this performance is free of over saturation and offers smooth control law.

[3] V. Parra-Vega, J.D. Fierro-Rojas, A. EspinosaRomero, Uncalibrated Sliding Mode Visual Servoing of Uncertain Robot Manipulators, 2003 IEEE International Conference on Robotics and Automation, May 12 - 17, Taipei, Taiwan.

[4] Hutchinson, S., Hager, G.D., and Corke, P.I., A Tutorial on Visual Servo Control, Trans. on Robotics and Automation, 12:651-670, 1996.

[5] W.E. Dixon, E. zergeroglu, Y. fang. and D.M. Dawson, Object Tracking by a Robot Manipulator: Robust Cooperative Visual Servoing Approach, Proceeding of the 2002 IEEE International Conference on Robotics \& Automation, 211-216

[6] Y. Shen, G.Xiang, Y.H. Liu, K. Li, Uncalibrated visual Servoing of Planar Robots, Proceeding of the 2002 IEEE International Conference on Robotics \& Automation, Washinton, D.C., 580-585.

[7] E. Gortcheva, R. Garrido, E. González and A. Carvallo Predicting a moving object position for visual serving: theory and experiments, International Journal of Adaptive Control and Signal Processing. 2001; 15:377-392.

[8] P. Anandan, "Computing dense displacement fields with confidence measures in scenes containing occlusion" Proceedings of SPIE, Inteligent Robots an Computer Vision, November 5-8. 1984.

[9] Personal Communication with M.Sc. Jorge Fierro R. jfierro@mail.cinvestav.mx.

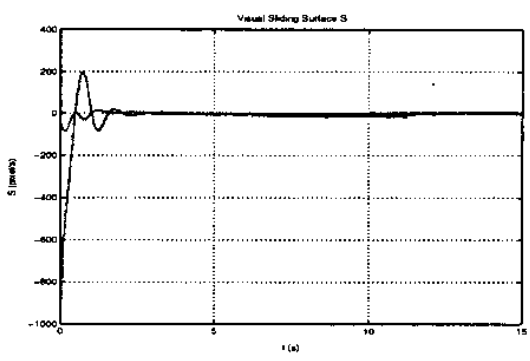

Fig. 10. Visual sliding surface behavior. The convergence of the surface is stable.

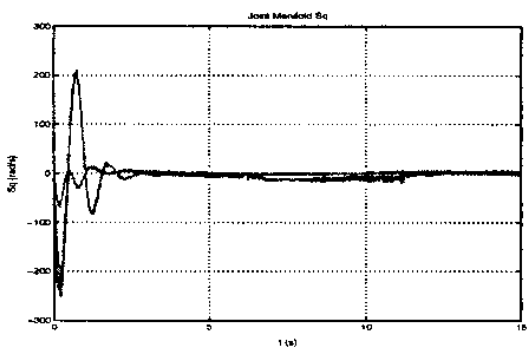

Fig. 11. The extended joint error behavior is similar to the visual sliding surface.

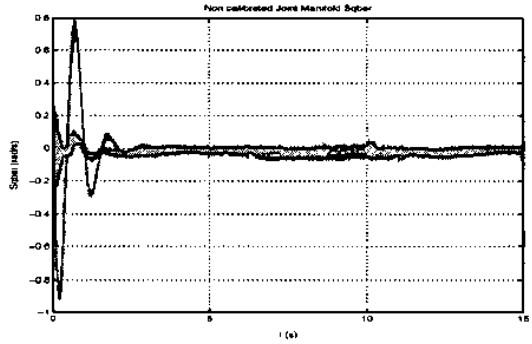

Fig. 12. The non calibrated extended joint error shows convergence even when uncertainty of robot dynamics and visual parameters is present.

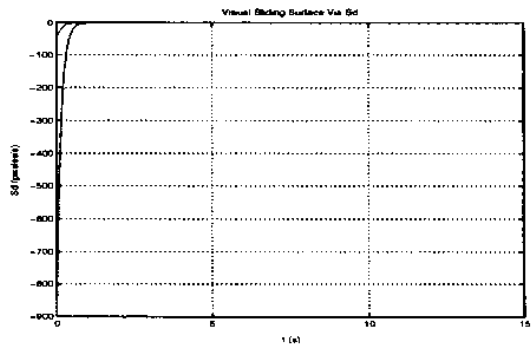

Fig. 13. The visual stiding surface vias behavior is exponential and its performance depends on constant $\kappa$. 